\author{
Military Technical College \\ Kobry El-Kobbah, \\ Cairo, Egypt.
}

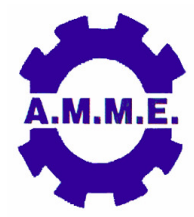
$15^{\text {th }}$ International Conference on Applied Mechanics and Mechanical Engineering.

\title{
OPTIMUM CHOICE OF GAS UNIT GEOMETRICAL CHARACTERISTICS TO ENHANCE ITS EFFICIENCY
}

\author{
M. S. Abo Elkhair ${ }^{*}$
}

\begin{abstract}
Automatic weapons are considered as an internal combustion engine, where the driving energy is supplied from the burning of the smokeless propellant charge, it burns producing tremendous power of hot gases. This power is extensively used in driving of projectile through the barrel, and the motion of weapon mechanisms that leads to the full automation of weapon functions.

In order to achieve the second goal of gases usage, a constructional arrangement is fixed on weapon barrel, this arrangement allows gases to branch and drive the main moving weapon component, this component is known as the breech block carrier [1]. The motion of which is responsible of moving all other mechanisms in automatic weapon.

The regulations and controlling over the output from the gas unit are affected basically by the changes of the geometrical characteristics of the gas unit. In this work, the variations of these geometrical parameters of the gas unit and their reflections on its efficiency have been studied. The present study facilitates the choice of effective parameters specially when designing a new weapon or modifying the power of an existing weapon.
\end{abstract}

* Egyptian armed forces 


\section{INTRDUCTION}

Upon classifying the automatic weapons according to the way of drive of their mechanisms; the Gas Operated System; (GOS) comes to the top of all classes; fig.1 shows a schematic diagram of a weapon drive by gas operated system. This preference stands on their possessing a wide range of control parameters over the impulse of gases, these gases are allowed to branch from the main stream of gases flowing in the barrel bore supplying the moving parts by the required energy for their movements. Similarly; the Recoil Operated System; (ROS) relies on the use of accelerators or recoil increasers when it is required to increase the momentum of the main recoiling part.

In the corresponding figure a simplified scheme of the moving parts of the GOS before and after firing is presented having:

- Gas vent.

- Piston head.

- Piston rod.

- Breech block (b.b).

- Breech block carrier (b.b.c.).

- 6- Return spring.

It is worth to mention that the gas operated system allows the wide control over the power of fire in automatic small arms (ASA). The following scheme of
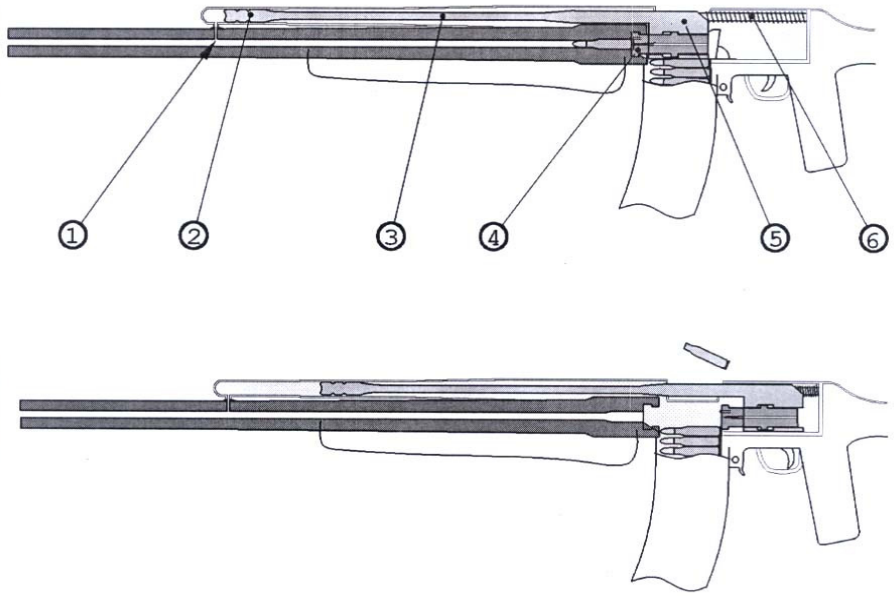

Fig. 1. Constructional arrangements of a weapon driving by GOS

gas unit shown in Fig.2 presents the controlling geometrical parameters through which the control over the efficiency of gas unit can be achieved. Consequently, the effect of these parameters on the efficiency of gas unit (GU) will be studied through the changes in: "the area of gas vent, the piston head diameter, the initial volume of gas chamber, and the unit controlling the outlet of gases from the gas chamber".

Therefore; it is necessary to design the gas unit such that the design parameters satisfies the optimum usage of branching gases, i.e. with minimum loss of gasses momentum and maximum value of GU efficiency, this task is very difficult owing to the large number of factors influencing the course of pressure in gas chamber[8, 9]. Let us quote some additional important factors:

1. Pressure value of powder gases in barrel at vent position

2. The efficiency of gas unit which is affected by:

a. Time corresponding to the end of burning of powder gases

b. Influence of muzzle devices (Muzzle brake, flash dampers... etc)

c. Place of the gas vent on barrel.

d. Form and dimensions of gas passages, gas chamber.

e. Annular clearance between piston head and cylinder guide. 


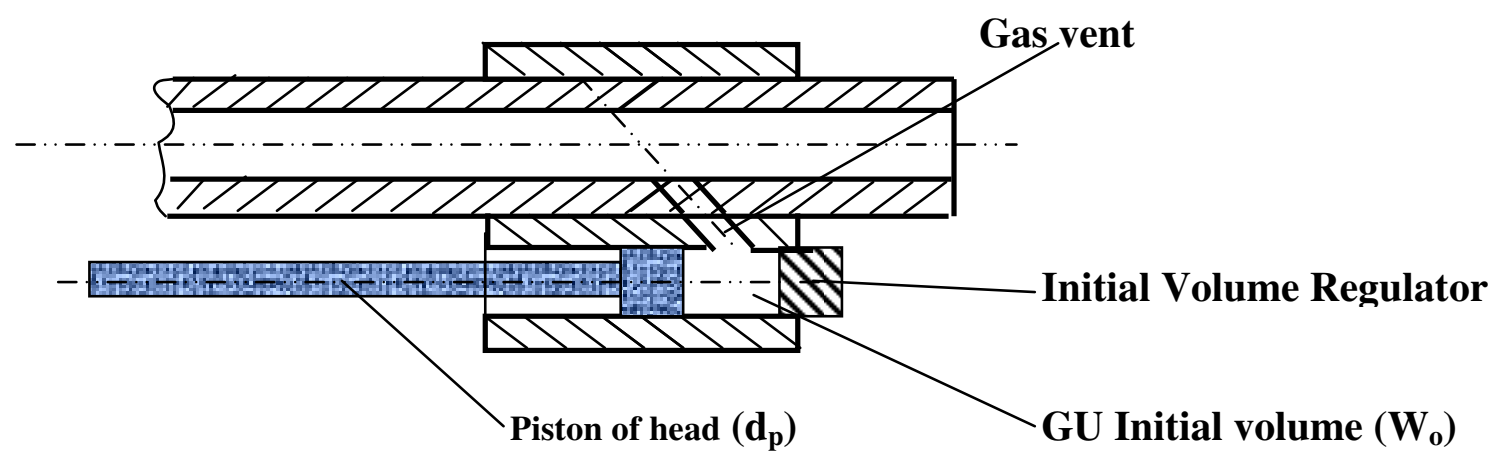

Fig. 2. Schematic diagram of gas unit showing geometrical characteristics.

f. Velocity of flowing powder gases along the bore.

g. Form of piston front part and its diameter.

h. Masses of moving parts connected with piston.

The majority of these factors can be included into calculations through empirical formulae based on experimental results. Then using the combination between experimental and theoretical results makes it possible to reach acceptable methods for determination of values necessary for construction and design of gas unit. Let us carry out the solution of two basic types of gas chambers. The first type which is presented schematically by (Fig.3-a); is in its substance a cylinder attached to the barrel and inside which there is a moving piston connected by a push rod to the movable recoiling parts.

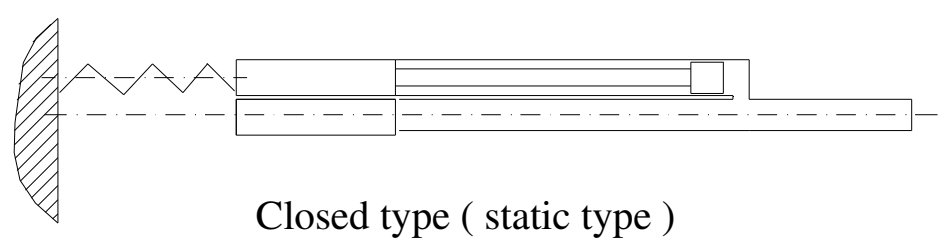

Closed type ( static type )

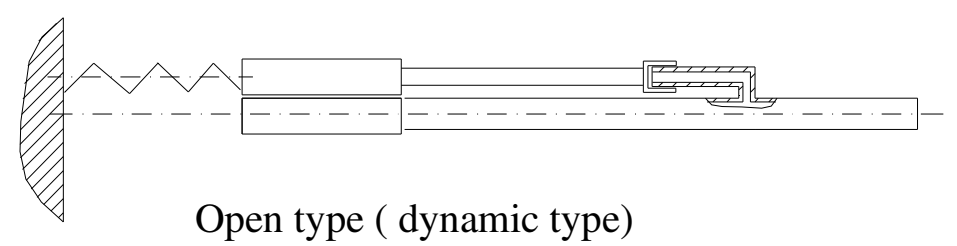

Fig. 3. Simplified scheme of closed type gas unit(a), and open type (b).

This system is well established in locked weapons with fixed barrel. After firing, the powder gases pass through the gas vent; fill the gas chamber and push the piston group backward; supplying the recoiling parts with the required energy to secure their automatic function. From constructional point of view, gas chamber constructions are divided into open or closed gas chamber; as shown in fig.3. At the same time; the walls of the cylinder serve as a guide way to the piston, and controls 
the leakage of gases; this type is commonly known as the closed type gas chamber. The clearance between piston and gas cylinder is relatively small; this causes a noticeable control over the leak of gases, which leads to the increase in the efficiency of gases performance if compared with the open one. On the other hand; this tightness creates the pumping action that resists the motion of moving parts in both directions.

While the other type shown in (fig.3-b); the gas chamber extension cylinder is built covering the piston head only, and is well known as open type gas chamber. It is characterized by its relatively large annular clearance between piston head and gas tube; this is intentionally made to secure free entrance of piston cup when reaching the extreme front position. Even though; this type is mostly recommended but after performing slight modifications to cure relatively the problem of quick escape of gases, on other hand it solves the pumping effect which takes place in the closed type.

Early investigations [8,9] support the idea of increasing the tightness in case of closed type within a short track of piston movement about (1.5:2) $d_{p}$ where $d_{p}$ is the diameter of piston head, after which small exhaust openings are created along the enlarged passage of piston external guiding in order to prevent the resisting effect of pumping action. This explains the reliability of the semi closed gas chamber [8]; the majority of ASA is adopting this technique. Keeping in mind that the main advantage of the GOS; in driving weapon components of ASA is that it allows wide controlling parameters for regulation of the intensity of impulse of gases for many purposes.

\section{BASIS OF OPTIMUM CHOICE OF GAS VENT POSITION}

Let us start adopting the modified type of gas chamber. Assuming that the momentum of breach block carrier is necessary for achieving the required rate of fire, it must be proportion to the impulse of powder gases acting on the main moving parts during time through which the bullet passes the communication channel to the end of supplementary effect of powder gases. Coefficient of proportionality will depend on construction of gas chamber and it varies with the change in the cross section of the communication channel. Considering the applied equation of gas flow $[3,5]$ uses the equations derived for the momentum of the whole system and represented by [8]:

$$
M_{R P}=2 k\left(\frac{2}{k+1}\right)^{\frac{k}{k-1}} A \phi \int_{o}^{t_{v}+T} p . S . d t
$$

Where $M_{R P}$ is the momentum of acting gases on recoiling parts, $k$ is the ratio of specific heat, $A=S_{o} / S$ is the ratio of cross-section area of gas vent to barrel crosssection, $\phi \quad$ Empirical coefficient depending on the type of gas expansion chamber and the value of the ratio $(A), t_{v}$ is the time of bullet travel from the gas vent to the 
muzzle, $p$ is the pressure of gases inside the gas unit chamber and $T$ is the total time of additional action of powder gases.

The integral $\int_{0}^{t_{v}+T} P . S . d t$ represents the change in projectile momentum from the moments of passing the gas vent position till the end of action of add supplementary effect of powder gases. While the change in projectile momentum from communication channel to muzzle point $\mathrm{M}_{\mathrm{p}}$ can be expressed by:

$$
\mathrm{M}_{\mathrm{p}}=\frac{\varphi q}{g} \cdot\left(v_{o}-v_{k}\right)
$$

Where $\varphi$ is the coefficient of fictitiousness of the projectile, $q$ is the projectile weight, $v_{0}$ is the muzzle velocity of projectile and $v_{k}$ is the projectile velocity at the position of gas vent

The impulse of gases due to the supplementary effect can be determined considering that the barrel bottom is affected by the pressure of escaping gases $R_{1}$; and that the reaction of escaping gases $G_{\text {sec }}$ produces $R_{2}$ :

$$
R_{1}=P_{k} \cdot S=\left(\frac{2}{k+1}\right)^{\frac{k}{k-1}} \cdot P \cdot S
$$

and

$$
R_{2}=\frac{G_{\mathrm{sec}}}{g} \cdot v_{k}
$$

Where $\mathrm{P}$ is the pressure of gases inside the barrel corresponding to the gas vent and $P_{k}$ is the pressure of gases inside the gas unit expansion cylinder.

The total effect of force of gases at barrel bottom is therefore $[2,4]$ :

$$
\mathrm{R}=\mathrm{R}_{1}+\mathrm{R}_{2}
$$

Consequently; the total affecting forces $\mathrm{R}$ acting on the face of recoiling parts during time of supplementary effect of gases causes the total impulse (I) where

$$
I=\int_{0}^{t_{v}+T} R \cdot d t
$$

When expressing equations for $R_{1}$ and $R_{2}$ using laws of incompressible flow of gases $[6,7]$, and substituting by the integral value of $(R)$ obtained from equation $(6)$, their Integration leads to the required impulse of gases (I), this integral considers the effect of gases from gas vent till the end of supplementary effect of powder gases AAG leading to the following relation: 


$$
I=\frac{1}{k+1}\left(\frac{2}{k+1}\right)^{\frac{k}{k-1}} \cdot \frac{\beta \cdot \omega}{g} \cdot v_{p}
$$

The expression $\frac{1}{k+1}\left(\frac{2}{k+1}\right)^{\frac{k}{k-1}}=(0.28: 0.24) \quad$ (assuming $\mathrm{k}=1.2: 1.3$ )

And the coefficient $\quad \beta=\left(\frac{2}{1+k}\right)^{2 / 3}\left(\frac{k+1}{k}\right)\left(\frac{1}{v_{0}}\right)\left(\sqrt{\frac{k p_{z}}{\gamma_{z}}}\right)$

Where $\beta$ is the coefficient of supplementary effect of gases [8], $p_{z}$ is the pressure of gases at muzzle point, $\mathrm{V}_{z}$ is the density of powder gases and $v_{0}$ is the muzzle velocity of projectile.

Substituting by the selected value of $\mathrm{k}$; the total momentum of gases acting on recoiling parts can be expressed in the form:

$$
M_{R P}=2 k\left(\frac{2}{k+1}\right)^{\frac{k}{k-1}} A . \phi\left[\frac{\varphi q}{g}\left(v_{o}-v_{k}\right)+0.28 \frac{\beta \omega}{g} V_{m}\right]
$$

where $V_{m}=V_{o}$ is the muzzle velocity of projectile and $M_{R P}$ is the total momentum of gases acting on recoiling parts.

Table 1. Values of fictive coefficient $(A \phi)$ as a function of ratio of cross-section area of gas vent to barrel cross-section $(A)$ :

\begin{tabular}{|c|c|}
\hline$A$ & $\phi$ \\
\hline 0.408 & 2.63 \\
0.260 & 4.0 \\
0.182 & 5.2 \\
0.147 & 6.1 \\
0.133 & 6.6 \\
0.096 & 8.3 \\
\hline
\end{tabular}

The formula in equation (8) becomes imprecise as far as the location of gas unit approaches the vicinity of muzzle [8,9]. The same effect takes place when utilizing branched gases by a tube rather often as presented in weapon gas vents.

When calculating the diameter of gas vent; we must take care that no interruption of bore rifling should take place. For this reason, when obtaining a greater diameter of the channel and having no possibility of change by shifting the place of gas unit closer to the chamber; then it is preferred making two or more lateral openings channels [8]. When intending to utilize this solution also for determination of conditions with the closed type gas chamber; we can replace the magnitude $\phi$ by value $\phi^{\prime}$ where: 


$$
\phi^{\prime}=\delta \phi
$$

And $\delta$ is the correction factor depending on gas unit type $[\delta=1.3: 1.4]$.

Hence, the above discussions lead to the optimum choice of gas vent position on the bases that it delivers the required momentum to the moving parts in order to satisfy the requirements of the power of fire for a specific automatic weapon.

\section{EMPIRICAL DETERMINATION OF GAS UNIT EFFICIENCY $\left(\eta_{K}\right)$}

The efficiency of gas unit is a vital parameter which evaluates its performance; it directly depends on the impulse of branching gases. Prof. Mamontove had handled this problem using the empirical formulae deduced from extensive firings with different calibers of automatic weapons. In order to formulate the effect of gas unit efficiency, it is recommended to study how the impulse of gases is determined [8,9]. For determination of specific impulse of gases in gas unit $\left(i_{k}\right)$ empirically, the following formula is used:

$$
i_{k}=i_{0} \cdot \eta_{k}
$$

where $\boldsymbol{i}_{o}$ is the total impulse of gases in the barrel bore from the vent moment till the end of discharge of gases and $\eta_{k}$ is the efficiency of gas unit.

$$
i_{0}=\frac{p_{v}+p_{z}}{2} t_{v}+\frac{p_{z}}{A}
$$

where $P_{v}$ is the pressure of powder gases inside the barrel corresponding to the point of gas vent, $P_{z}$ is the pressure of powder gases inside the barrel corresponding to the muzzle point, $A$ is the coefficient of additional action of powder gases and $t_{v}$ is the time taken by projectile from the gas vent till the muzzle.

$$
\begin{aligned}
& t_{k}=(1.1: 1.2)\left(t_{v}+T\right) \\
& \eta_{k}=\eta_{0} \cdot \nu_{0} \cdot v_{q} \cdot \nu_{q_{0}}
\end{aligned}
$$

All the values of the above functions are tabulated in terms of different parameters $\left(\sigma_{0}, \sigma_{\pi}, \sigma_{\Delta}, \sigma_{\mathrm{q}}\right)$ which in turn affects the performance and efficiency of gas unit such as: 


$$
\left.\begin{array}{l}
\eta_{0}=f\left(\boldsymbol{\sigma}_{\pi}, \boldsymbol{\sigma}_{\Delta}\right) \\
\boldsymbol{\nu}_{0}=f\left(\boldsymbol{\sigma}_{\pi}, \boldsymbol{\sigma}_{0}\right) \\
\boldsymbol{\nu}_{q}=f\left(\boldsymbol{\sigma}_{\pi}, \boldsymbol{\sigma}_{q}\right) \\
\boldsymbol{\nu}_{q_{0}}=f\left(\boldsymbol{\sigma}_{q}, \boldsymbol{\sigma}_{0}\right)
\end{array}\right\}
$$

The value of the above parametric functions can be obtained from:

$$
\begin{gathered}
\sigma_{\pi}=S_{p} / S_{0} \text { and } \sigma_{\Delta}=\Delta S_{p} / S_{0} \\
\sigma_{q}=M / S_{p} \text { and } \sigma_{o}=W_{o} / S_{0}
\end{gathered}
$$

where $S_{p}$ is the cross-sectional area of piston head, $S_{o}$ is the cross-sectional area of gas vent, $\Delta S_{p}$ is the annular clearance between piston head and ext. guide, $W_{o}$ is the initial volume of gas unit chamber and $M_{R P}$ is the mass of recoiling parts (breech system+ piston group+ spring).

It is worth mentioning that the functions mentioned above in equation (14) can be determined using tables of gas unit [8]. But in this work, a computer program has been created and tested as a substitute to Mamontove tables. The accuracy of results compared with Mamontove tables has proved more accuracy due to the advance in computing techniques.

\section{REMARKS ON MAMONTOVE'S APPROACH IN DETERMINING GU EFFICIENCY}

Due to the rareness of references in these topics, the available references $[8,9]$ show that Mamontove has performed a great job. In spite of being created a set of empirical formulae and tables that describe the variation of different parameters such as $\eta_{0}, \eta_{k}, v_{0}, v_{q}, v_{q o}$ their dependencies have considered most of the geometrical characteristics that describe the construction of gas unit, equations 15, 16, and 17 present this dependency. But on other hand; the weight of variation in gas unit efficiency sometimes doesn't reflect the affecting parameter, this is what has been discovered when following the comparative study and calculations of gas unit efficiency using Mamontove's approach [8]. Survey and analysis of the results will present this notice, while the recommendation suggested the way through which it will be possible to cure what has been considered as deficiency in this empirical approach.

\section{RESULTS AND ANALYSIS}

Using the powerful MathCad14; the changes in the efficiency of gas unit have been studied in different cases. As mentioned early, the geometry of gas unit plays the important role in affecting the values of Geometrical Functions (GF) mentioned in equations 16, 17. Since the empirical values of Mamontove's functions are extracted 
from tables as function of these GF, the work has dealt with the variation of the geometrical dimensions, consequently the resulted GF as well as their reflection on the efficiency of gas unit $(\eta)$.

Following are the results as sorted in groups as:

Geometrical functions variation due to $(\mathrm{dg})$ variation

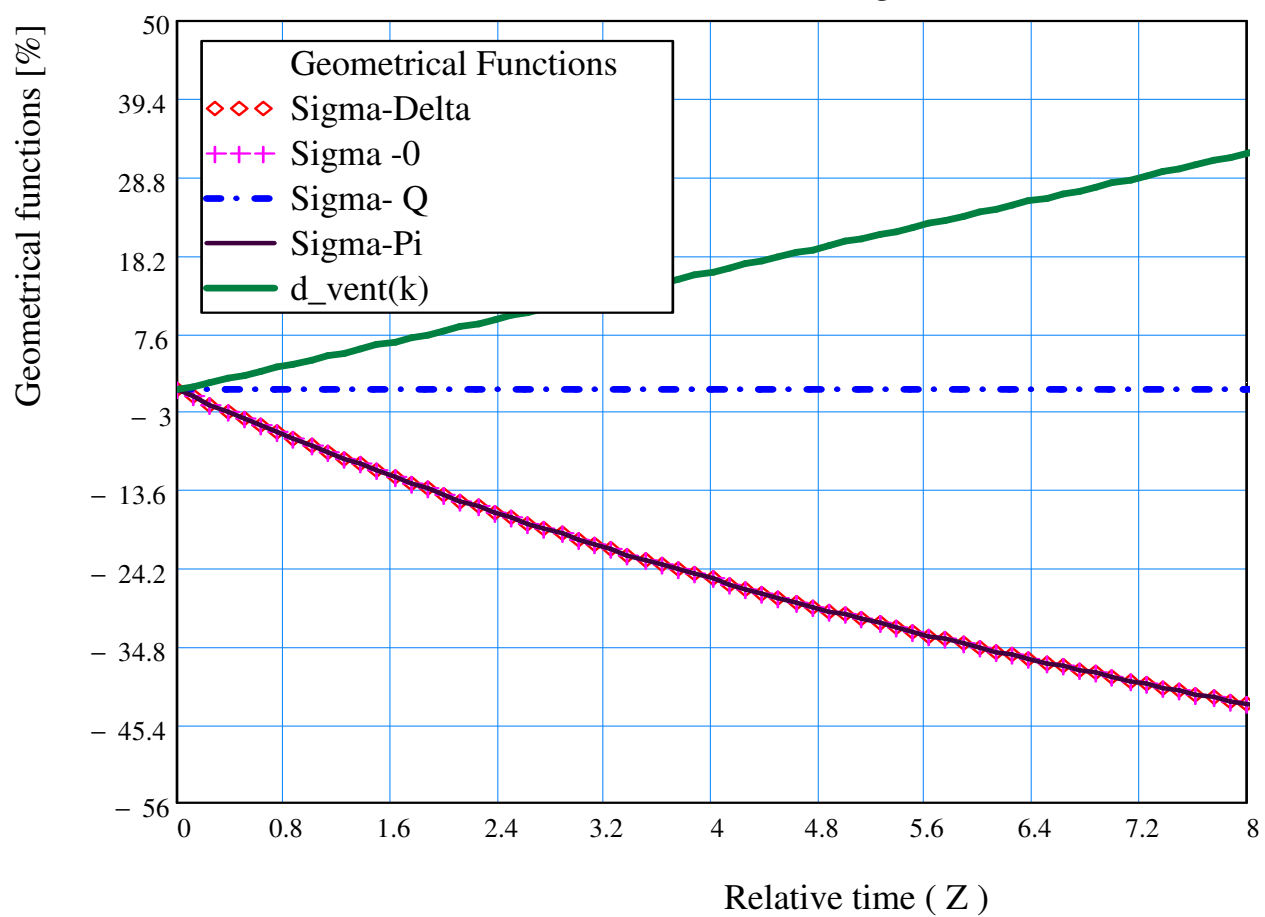

Fig. 4. Changes in geometrical characteristics due to (dg) variation.

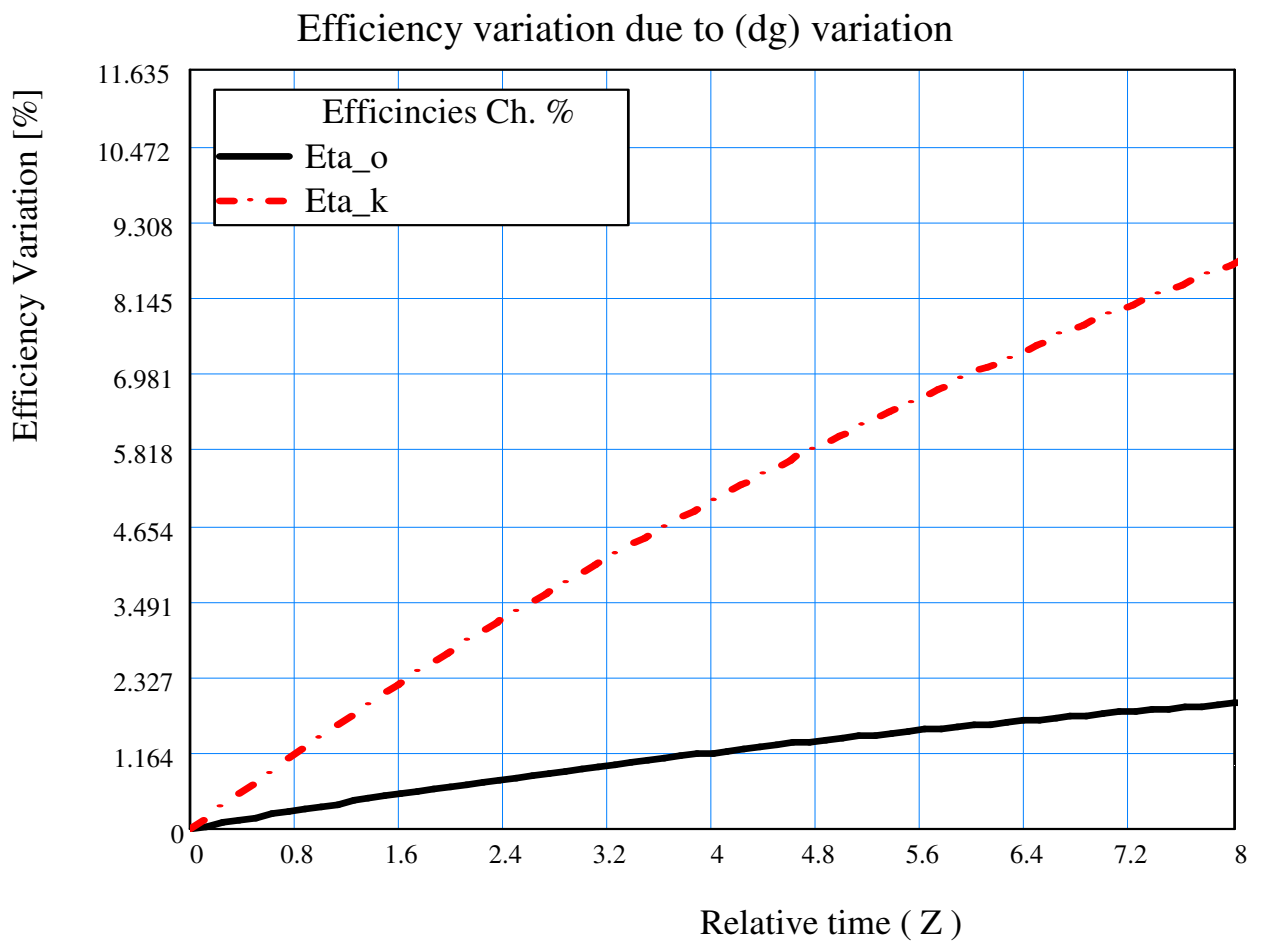

Fig. 5. Changes in GU efficiency due to (dg) variation. 
Geometrical functions variation due to (dp) variation

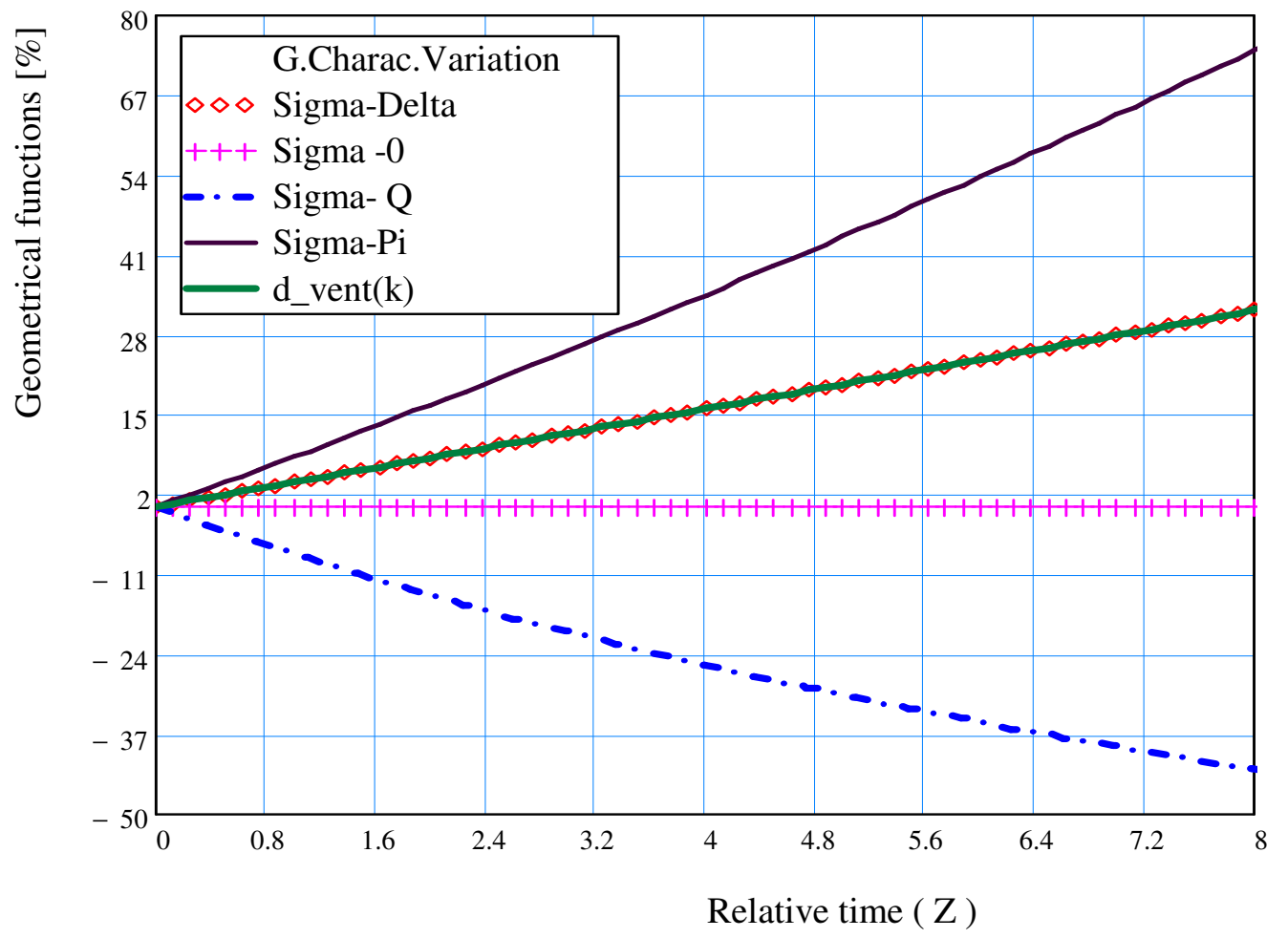

Fig. 6. Changes in geometrical characteristics due to $(\mathrm{dp})$ variation.

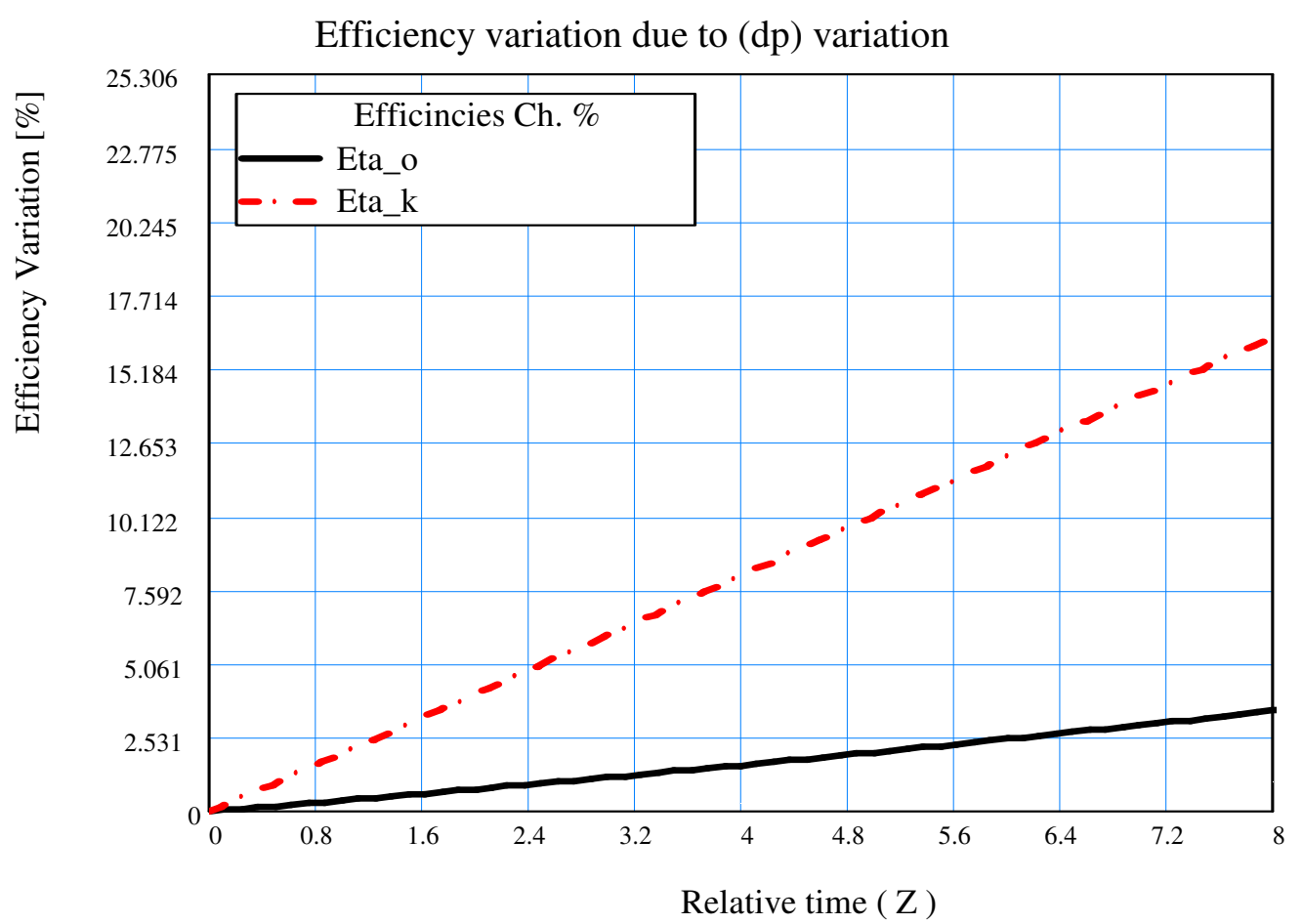

Fig. 7. Changes in GU efficiency due to (dp) variation. 
Geometrical functions variation due to changes in clearance

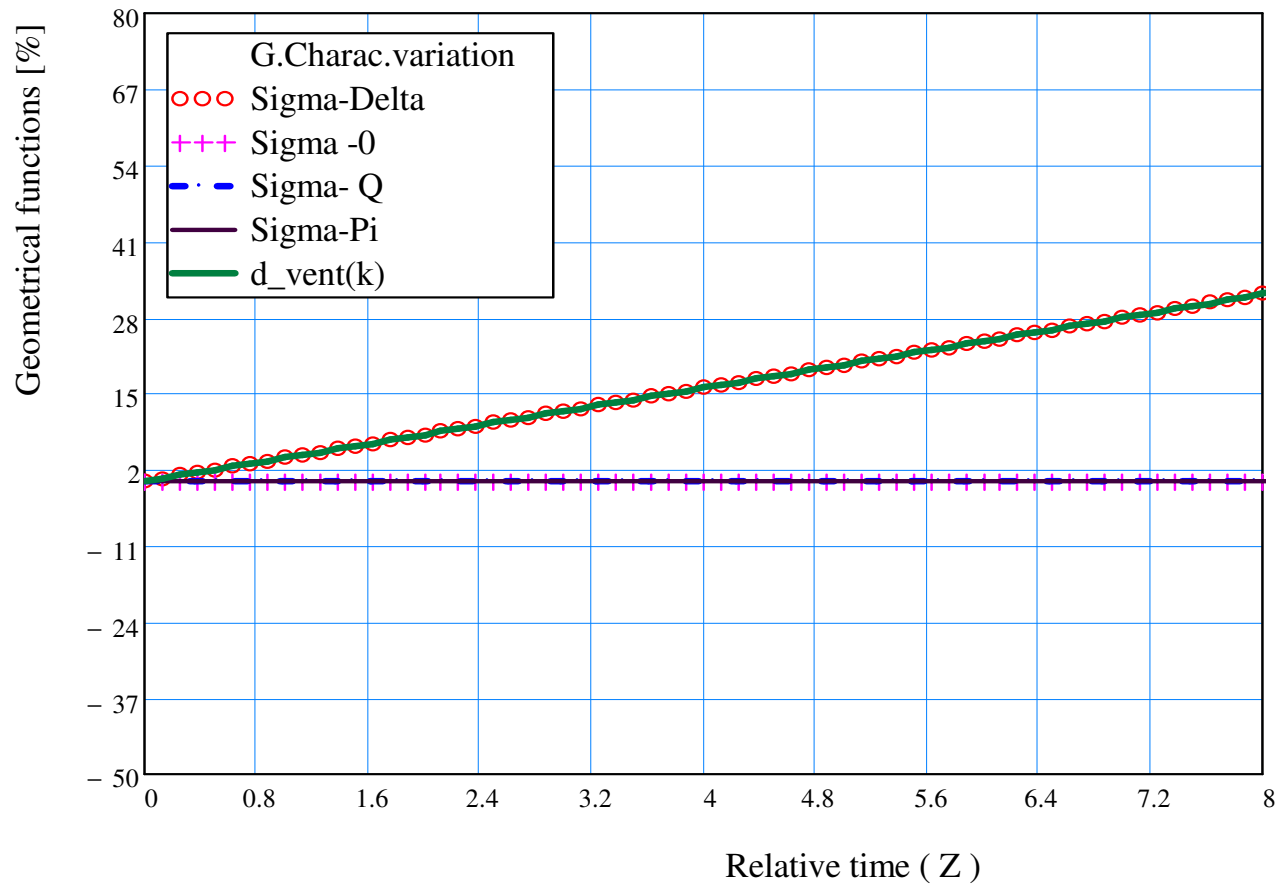

Fig. 8. Changes in geometrical characteristics due to $(\Delta \mathrm{dp})$ variation.

Efficiency variation due to diametric clearance variation

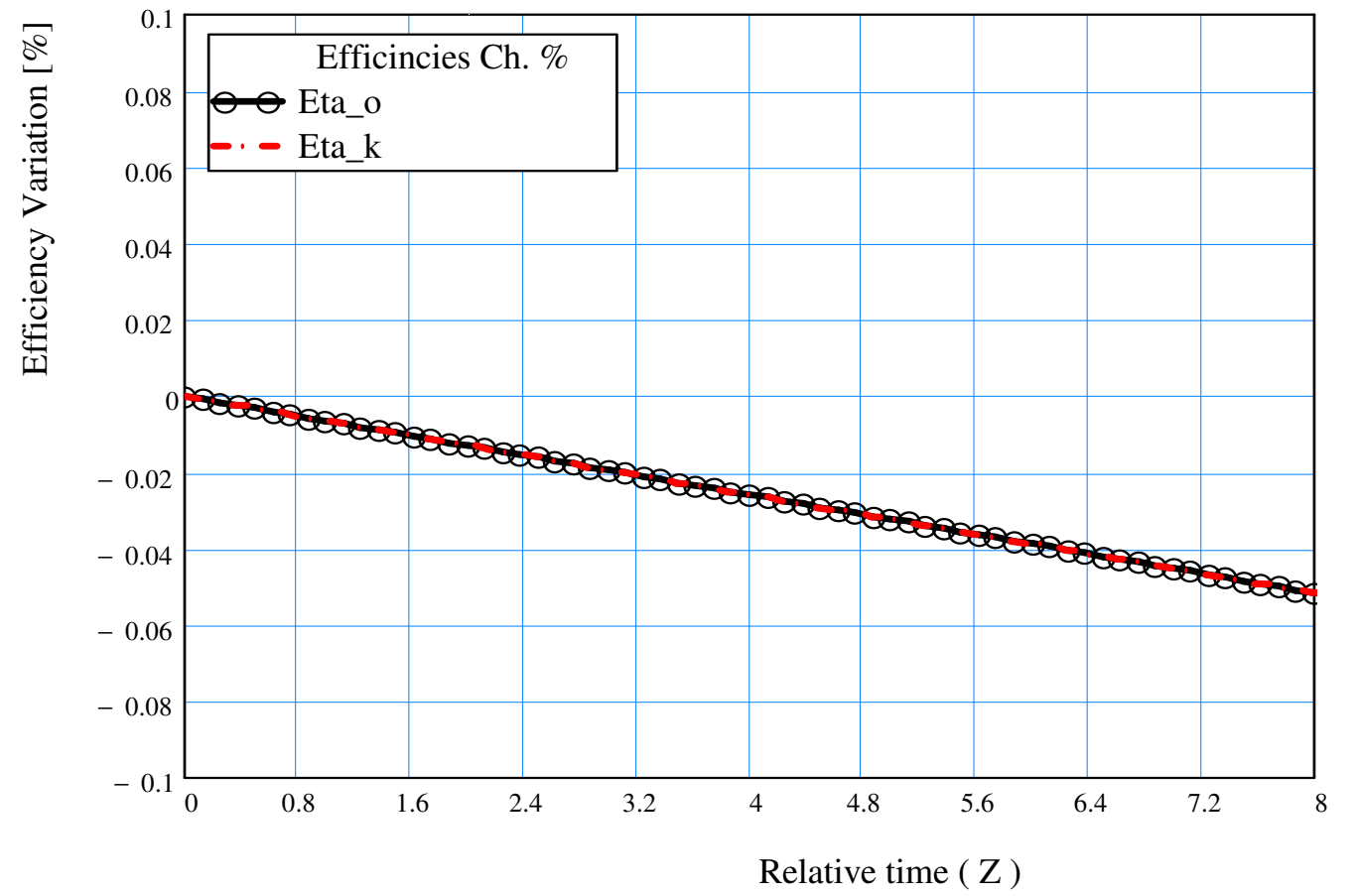

Fig. 9. Changes in G.U. efficiency due to increase in diametric clearance. 
Geometrical functions variation due to changes in (Wo)

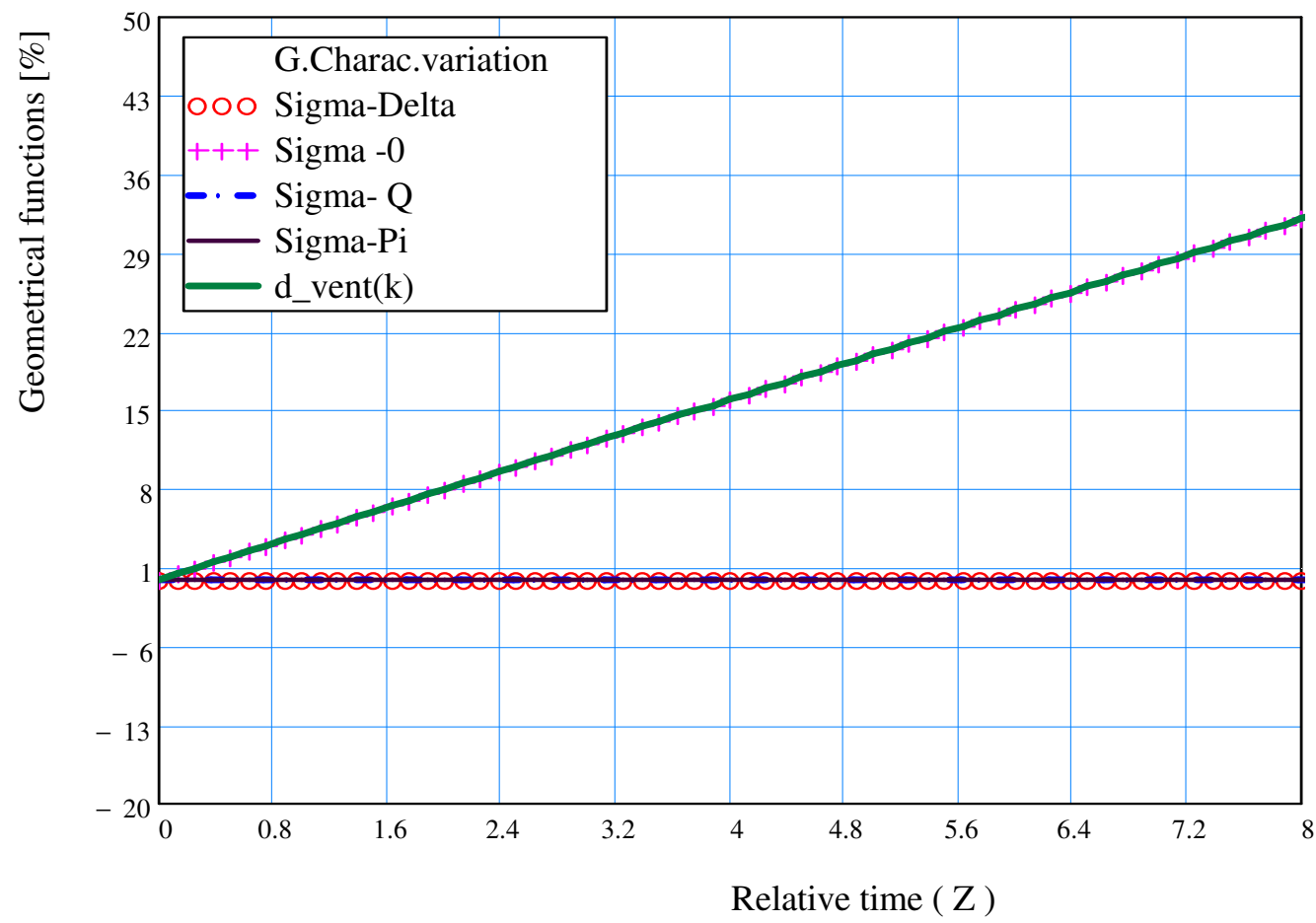

Fig.10. Changes in geometrical characteristics due to $\left(W_{0}\right)$ variation.

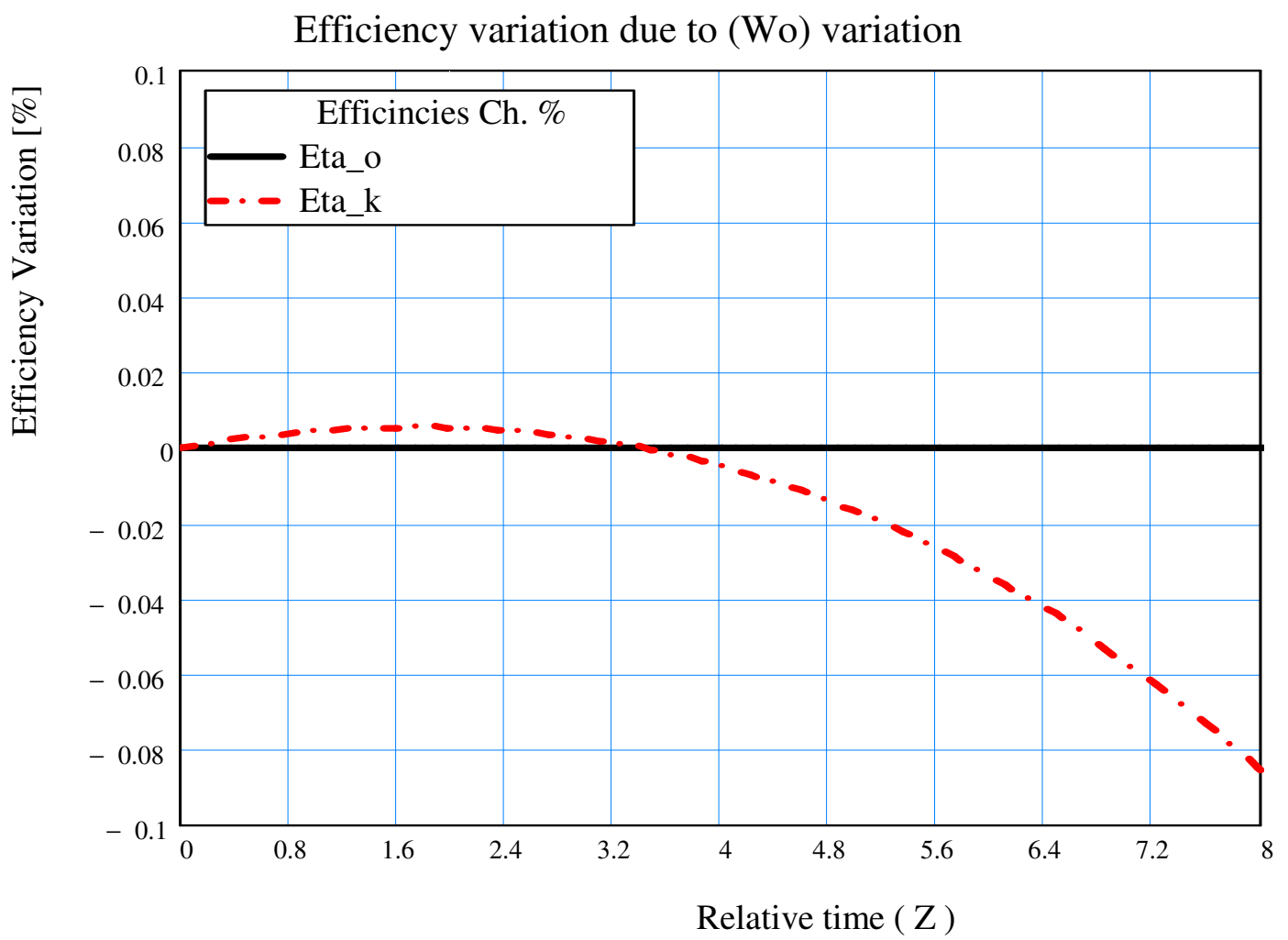

Fig. 11. Changes in GU efficiency due to variation in $\left(W_{0}\right)$. 


\section{CONCLUSIONS}

The analysis of results shown in the previous figures from fig. 4 to fig. 11 explains how the efficiency of gas unit is affected by the change in the gas unit parameters. These changes have been selected as a percentage of the change from the original dimensions of these aforementioned parameters, the upper limit of each parameter reaches $50 \%$ of its original value. These changes are also performed in 100 steps; each one doesn't exceed $1 \%$ to insure the accuracy of the changes.

The analysis of results could lead to the following facts:

- The change of the diameter of piston head is the most effective parameter when the design aims to increase the gas unit efficiency; the percent of change increases the efficiency of gas unit to $16.25 \%$ from the original value, which in turn affects the power of fire positively.

- The change in the diameter of gas vent comes secondly raising the efficiency to $8.56 \%$ from the original value.

- The changes in diametric clearance and initial volume of the expansion cylinder have negative effect on gas unit efficiency.

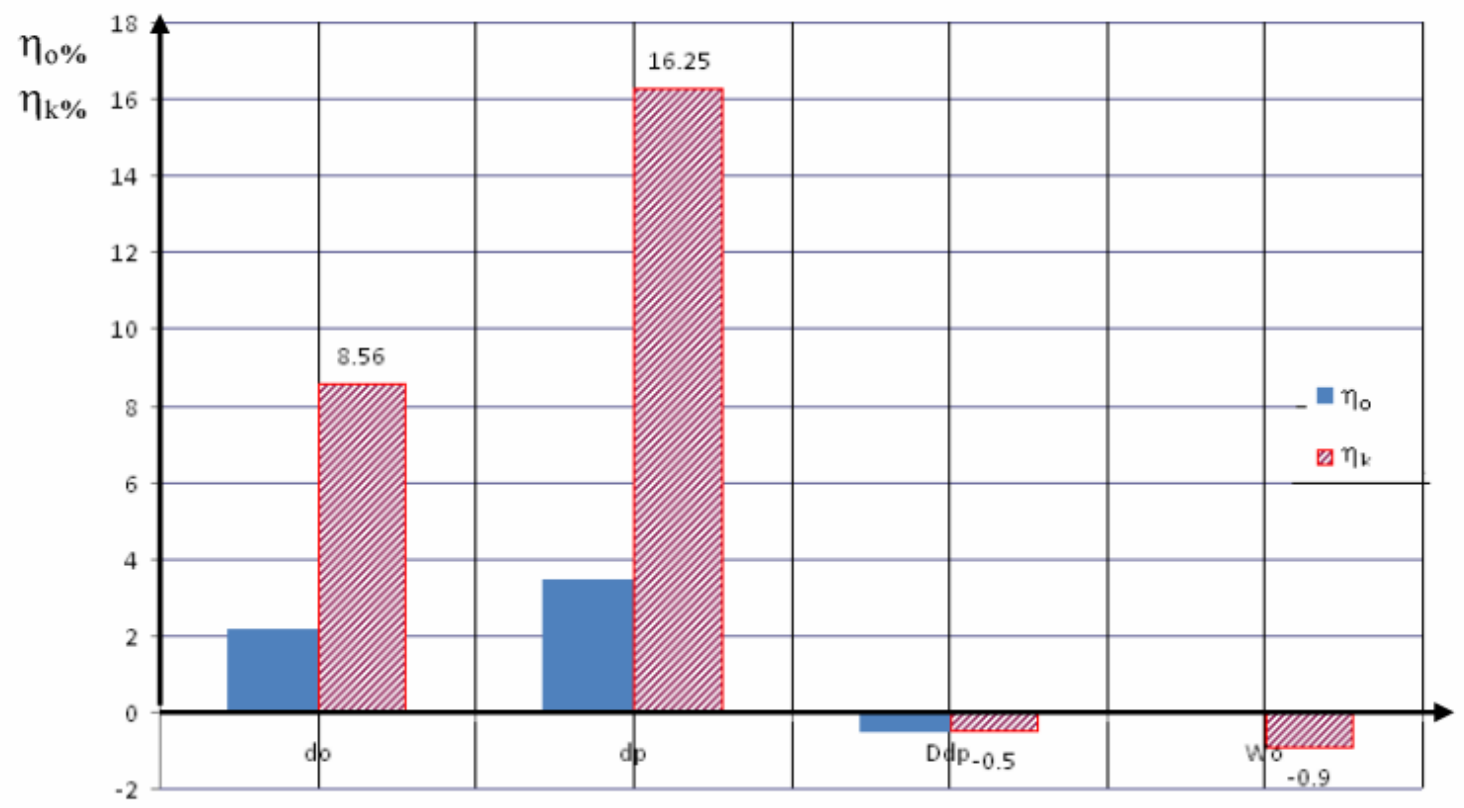

G.U.Parameter

Fig.12. Changes in GU efficiency due to $50 \%$ increase in gas unit parameters

As a conclusion, however the changes in piston head has the lead in improving the efficiency of gas unit, the combined effect with the increase of $W_{0}$ must be considered. In addition; the effect of reduction in momentum of gases behind the projectile must be considered when thinking in increasing the gas vent diameter to increase the efficiency of gas unit. Finally; the problem can be handled globally when the power of fire is needed to be optimized. 


\section{REFERENCES}

[1] Anon, "Construction of automatic small arms", MTC Printed Lectures No.239, Figure part, MTC, Cairo, Egypt (1960).

[2] Genick Bar-Meir, "Fundamentals of Compressible Fluid Mechanics", Amazon Press, USA (2008).

[3] John Anderson, "Modern Compressible Flow: With Historical Perspective", $2^{\text {nd }}$ ed., Amazon Press, USA, July (2002).

[4] M. Potter and D. C. Wiggert, "Fluid Mechanics", Schaum's Outlines, McGraw Hill Co., USA (2008).

[5] P. A. Thompson, "Compressible fluid dynamics - Advanced engineering services", Amazon Press, USA (1972).

[6] R.J. Prozan, L.R. Center, "A variation principle for compressible fluid mechanics: discussion of the one-dimensional theory" , National Aeronautics and Space Administration. USA (1982).

[7] S. J. Shamroth, W. R. Briley, "A viscous flow analysis for the tip vortex generation process", Langley Research Center, USA, (1979).

[8] Anon, "Theory and design of small arms ", MTC Printed Lectures No.40 Vol. 1, MTC, Cairo, Egypt, (1960).

[9] Anon."Theory and design of small arms ", MTC Printed Lectures No.40 Vol. 2, MTC, Cairo, Egypt, (1960). 